\title{
A LOW COST UNDERWATER TEST ENVIRONMENT
}

\author{
Joschka van der Lucht ${ }^{*}$, Michael Bleier, Andreas Nüchter \\ Informatics VII - Robotics and Telematics, Julius Maximilian University of Würzburg, Germany \\ \{joschka.lucht, michael.bleier, andreas.nuechter\}@uni-wuerzburg.de
}

Commission II

KEY WORDS: underwater, test environment, 3d laser scanning, low cost, 3d reconstruction, container

\begin{abstract}
:
In this paper we present the setup of a low cost underwater test environment. The employed materials and structures are described as well as the lessons learned during operations and experiments conducted in the water tank. We provide the bill of materials and a break-down of the costs involved in setting up the test site.

The goal was to build a low cost test environment for underwater applications, because our lab is around 40 minutes by car from the nearest lake. Also a natural lake is not an optimal location for first test and experiments, because the access to the water is difficult and logistics of equipment is time consuming. This work aims to describe what experiences we have gained during the time of construction. Which materials we used and what we would do differently next time. Furthermore, we describe the idea and process to place calibration markers inside the container and how the water thwarted our plan. We show that it is possible to realize a useful test environment for underwater experiments for less than $€ 20000$.
\end{abstract}

\section{MOTIVATION}

An underwater test environment near to the lab with the possibility to have the full control over the environment parameters, is necessary for carrying out comparable experiments. Our lab is around 40 minutes by car from the nearest lake and around 15 minutes to a river. However a natural lake or river is not an optimal location for first tests and experiments, because the access to the water is difficult and the environment variables, such as turbidity, are uncontrollable. Besides logistics of equipment is time consuming and not realizable without car. Therefore we build our own low cost test environment, depicted in Fig 1, for less than $€ 20000$.

This work aims to describe what experiences we have gained during the time of construction. Which materials we used and why we chose it. What we would do differently next time and which decisions were right. Furthermore, we describe the idea and process to place calibration markers inside the container and how the water thwarted our plan. We also report on our experiences so far and on our first experiments in the test environment. Last we show the composition of our first larger test object.

\section{CONSTRUCTION OF THE TEST ENVIRONMENT}

The container is a customized Roll-on/Roll-off container, build by Bohemia Behälter ${ }^{1}$, with a size of $7 \mathrm{~m} \mathrm{x} 2.4 \mathrm{~m} \mathrm{x} 2.4 \mathrm{~m}$, which results in a volume of up to 400001 of water. In order to prevent children, foliage and animals from falling into the container, we choose a solid metal lid that can be opened with a winch. Bohemia Behälter had an great customer service who engineered the container especially for our requirements. Moreover they was the only company which was able to build us the container without cross struts, as they would have hindered our experiments. The reason to choose a container instead of building a concrete pool is foremost cost-effectiveness. This custom made Roll-on/Rolloff container only costs $€ 7253$ and another major advantage is the mobility. It is easy to pick up the container with a truck and

\footnotetext{
${ }^{1}$ https://www.bohemia-behaelter.de
}

drive it to another place, for example, for demos. It is also easier to acquire permits for a non-permanent construction project because deconstruction and relocation of the container is possible with small efforts. Just a graveled place and electrical power is required. In addition, a water connection in the vicinity is recommended. In our case we used a hydrant $200 \mathrm{~m}$ away. With the help of the fire fighter school of Würzburg and their hoses we were able to fill the tank completely with water in about 40 minutes.

To keep the water clear we use a Oase BioTec ScreenMatic ${ }^{2}$ Set $90000^{2}$ system. This biological pond filter system does not require any additional chemicals. All bacteria and seaweed are killed by a UV lamp and afterwards filtered by sponges. Since we often get into the water during experiments and to protect our equipment, it should contain as few chemicals as possible. Moreover, the used filter and pump system is certified that we can safely work in the water during operation. Furthermore, the costs for ongoing operation and the maintenance costs should be as low as possible. Typical pool filter systems are use only sand to filter suspended particles out of the water and need additional chemicals to keep the water clean. There are also filter systems that filter the dirt out of the water with a fabric, but these have very high maintenance costs.

In order to be able to work well in the container, a scaffolding was placed in a U-shape around three sides of the container. The height of the platform is $1.8 \mathrm{~m}$, so we can reach easily over the edge of the container into the water, but the edge is still high enough to prevent falling into it. Next to the scaffolding we mounted a special stairway to climb up to scaffolding and get equipment on top of it. Because we mainly work with camera and light based systems, it is to be expected that some experiments are also carried out in the dark. For this reason we mounted bright LED headlights for lighting outside and special underwater lights in the container. Since the underwater lights should not pose any danger when working in the water, we used more expensive, but underwater certified lamps from Oase ${ }^{2}$. This, so called Oase LunAqua Power LED XL 4000 Wide Flood, led lights have $1320 \mathrm{~lm}$

\footnotetext{
2 https://oase-teichbau.de
} 


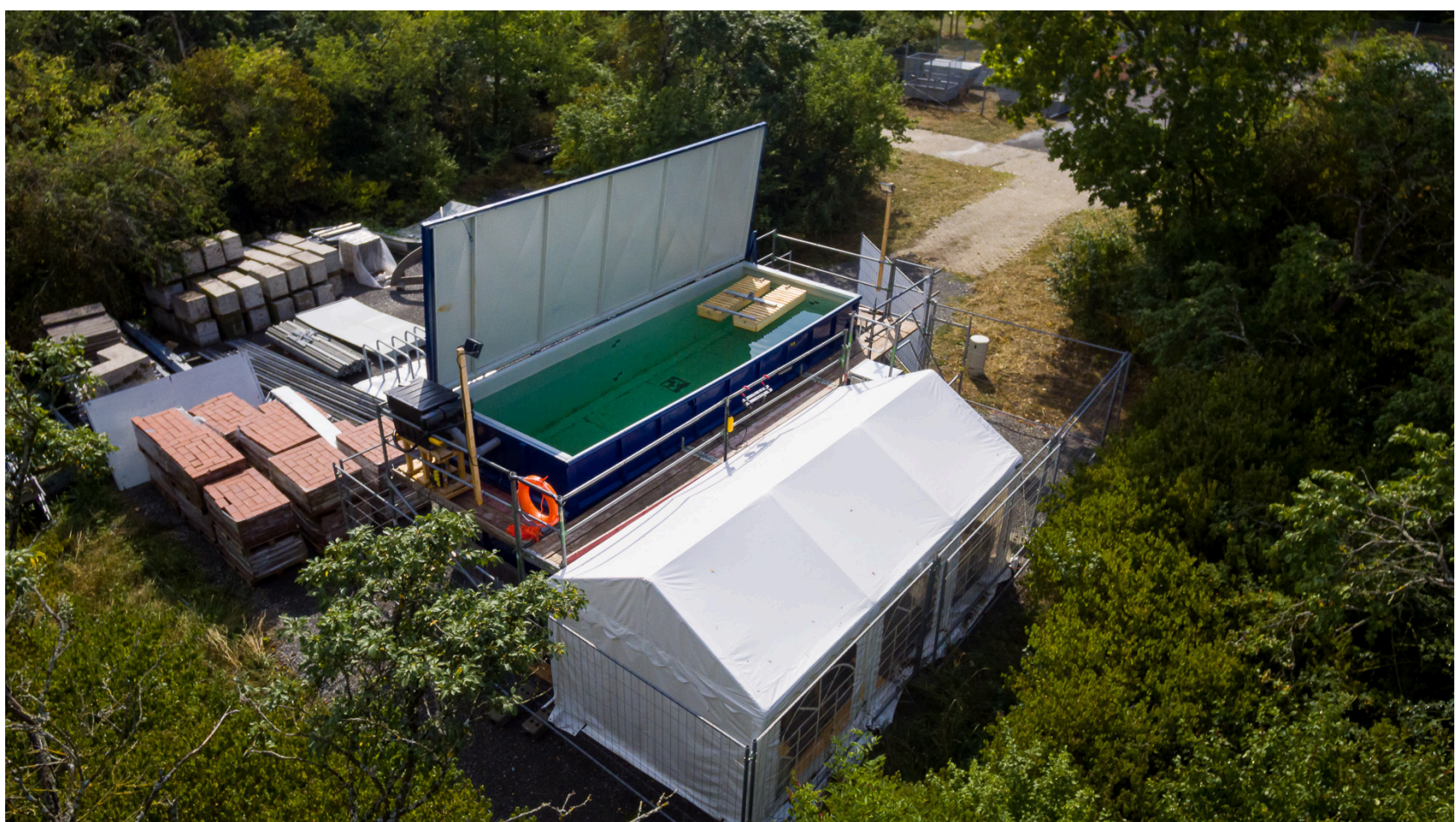

Figure 1. Overview of our test environment. The $7 \mathrm{~m} \mathrm{x} 2.4 \mathrm{~m}$ x $2.4 \mathrm{~m}$ Roll-on/Roll-off container filled up with around 400001 of water, a tent to have a dry working place and the scaffolding around the container are visible.

luminosity at $4000 \mathrm{~K}$ color temperature and a beam angle of $44^{\circ}$. We used two of them, mounted on two stirrups so that they can be hung over the edge of the container at different positions in the water.

The inside color is neutral gray (RAL7035) because this is neutral background for camera based applications and outside we chose the blue of our university (RAL5003). On the ground we placed four $30 \mathrm{~cm} \times 30 \mathrm{~cm}$ AprilTags, seen on the floor in Fig. 2 left and Fig. 3. We also placed some tags on the walls, four at the front and four at the back and two at each side. The AprilTags are placed to enable using visual odometry and visual tracking during future experiments. The twelve tags on the sides are intended to allow reference measurements. Before the container was filled with water we measured the coordinates of the markers. First, we captured a 3D scan of the whole container using a Riegl VZ400, seen in Fig 2 right, the result is shown in the left image in Fig. 8. Afterwards we used an OptiTrack V120:Trio optical tracking system seen in Fig. 2, to determine the positions of all placed markers. In theory this provides good position accuracy, but after filling up the container with water, we realized that the container was dynamically deformed by the water pressure and thus all scans and measurements were rendered incorrect.

Right next to the container we placed a $6 \mathrm{~m} \times 3 \mathrm{~m}$ tent, this is also visible in Fig. 1 and Fig. 3, to store equipment and provide a dry workplace. The tent is also used for the electrical installation and the internet access point. In addition, we spent about $€ 1270$ for small parts, such as pipes, wood, screws, cables and the like. A breakdown of the costs incurred can be seen in Tab. 1, in total we spent about $€ 16222$. In the future, we are planning to install sensors to monitor the water temperature and the filter system, as well as a webcam for longtime experiments.

\section{FIST USAGE AND ADDITIONAL EQUIPMENT}

\subsection{Structured Light Underwater Laser Scanner}

The underwater $3 \mathrm{~d}$ data presented in this paper was captured with a self-built structured light underwater laser scanning system. We choose high power laser line projectors because of the high absorption of light especially in turbid water conditions. Moreover, when scanning in surface water ambient light from the sun is an issue. The laser projection needs to be bright enough, such that sufficient contrast from ambient illumination is achieved. High power lasers and high sensitivity cameras with large dynamic range mitigate these problems to some degree.

We choose a cross line laser pattern because this enables scanning with mostly unrestricted movement. Only the distance between the scanner and the object has to be kept in a certain range, because of field-of-view and focus restrictions.

The two laser planes are projected at an angle of $45 \mathrm{deg}$ with respect to the vertical camera axis. This way both projected laser lines have approximately the same baseline. However, the resulting baseline is reduced compared to the mounting distance of the camera and laser projector housings.

3.1.1 Underwater Scanner Hardware The developed structured light underwater laser scanner consists of two housings with flat port glass windows, one containing the camera and the other one the cross line laser projector. The system is depicted in the left image in Figure 4. The two housings are mounted on a $0.5 \mathrm{~m}$ long aluminum bar. Custom mounts for the housings were manufactured using 3D-printing. The camera housing is mounted at an angle of $30^{\circ}$ to the bar. On top, a larger housing with lithium polymer batteries is mounted, which allows up to 6 hours of scanning time. This housing also contains the motor control electronics and a network switch to connect the underwater scanner to the surface via an underwater cable. The scanner is placed on a robotic joint with slewing ring bearings and a 1:50 worm 

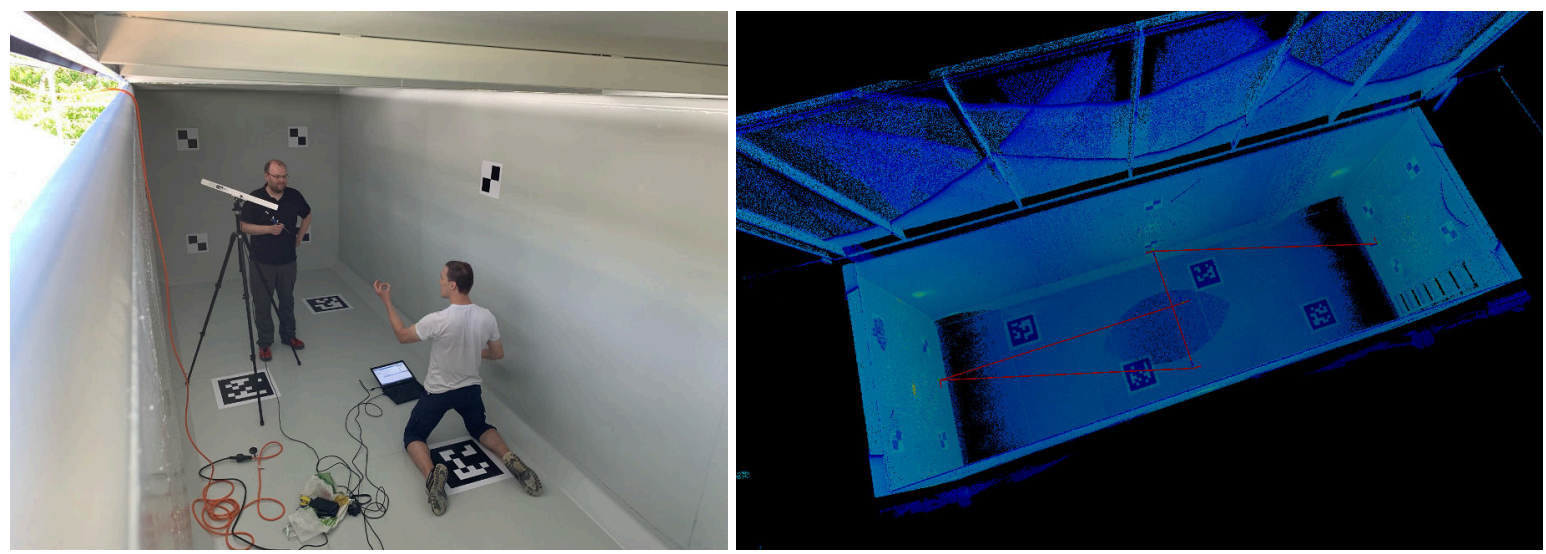

Figure 2. Left: Look inside the container without water. An OptiTrack V120:Trio system is used to determine the positions of the placed makers. Right: A point cloud of the container captured with a Riegl VZ-400 before the container was filled with water.
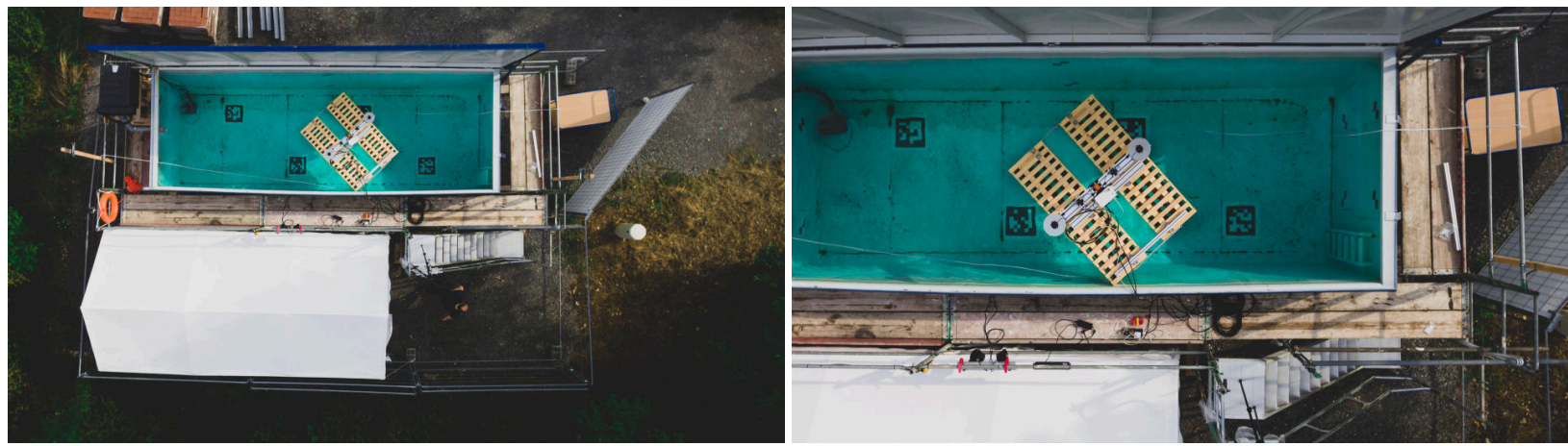

Figure 3. Top view of the water container. The placed AprilTags on the floor and an experimental setup with a raft can be seen.

gear, which is driven by a stepper motor. This allows rotating the scanner to capture $360 \mathrm{deg}$ scans. A magnetic encoder sealed in epoxide resin is used to measure the rotation angle of the scanner. All housings include embedded PCs with network interface. The Robot Operating System (ROS) is used as a middleware for sensor interfaces, logging and data processing. All embedded PCs are time-synchronized using Network Time Protocol (NTP) and a pulse-per-second signal. For synchronization of the camera with the laser projector a dedicated trigger pulse signal is used.

The right image in Figure 4 shows the electronics and optics components mounted inside the underwater housings. The camera assembly includes the lens with a focal length of $12.5 \mathrm{~mm}$. The camera is a FLIR Blackfly 2.3 Megapixel color camera with a 1/1.2" Sony Pregius IMX249 CMOS sensor. The image resolution is $1920 \times 1200$ pixels with $5.86 \mu \mathrm{m}$ pixel size and a maximum framerate of $41 \mathrm{fps}$. For image processing an embedded PC with an Intel Atom x5-Z8350 processor is included in the housing.

The cross line projector is constructed from Powell laser line optics, beam correction prisms and the laser diodes. The lasers are two $1 \mathrm{~W}$ green diode lasers with a wavelength of $525 \mathrm{~nm}$, which are mounted to an aluminum heat sink. The laser output power is controlled by two laser diode drivers, which can be adjusted via PWM signals generated by a microcontroller connected to an embedded PC. The two laser lines project a laser cross consisting of two perpendicular lines in the scene. The fan angle of the laser lines is $45 \mathrm{deg}$, which is reduced in water to approximately $32 \mathrm{deg}$. The total field of view is therefore $360 \mathrm{deg} \times 32 \mathrm{deg}$. The lasers are fired synchronized to the camera shutter using trigger pulse signals. An alternating firing order of the individual lasers is employed, such that each image captured by the camera includes only one of the two laser lines.
Fig. 7 left depicts the scanner mounted on a tripod and deployed for testing in our test environment. All components of the scanner are rated for $100 \mathrm{~m}$ water depth. The main components of the scanner, such as the underwater housings, cables and connectors are rated for more than $1000 \mathrm{~m}$ water depth.

\subsection{Test Structure}

In addition to the existing calibration pattern, depicted in Fig 7, consisting of two glass plates in a 90 degree angle, which are printed on the entire surface with ApriTags, we have built a large $1 \mathrm{~m} \times 1 \mathrm{~m} \times 2 \mathrm{~m}$ test object, depicted in Fig. 5. The goal was to create a structure, we can scan with our scanner mounted on an tripod and also scan from above mounted the scanner on a small ship for mobile mapping. For references and validation we acquired a point cloud of this object by an Riegl VZ-400, the result is depicted in Fig. 6. We used two types of pipes with different diameters. The main part was build with HT-pipes of $90 \mathrm{~mm}$ diameter and the two large pipes on the bottom of the structure have an diameter of $110 \mathrm{~mm}$. To ensure that the structure remains dimensionally stable, the pipes were mounted on a metal frame. One reason why we used this pipes is, that scanning pipes is an real requirement for many applications. Second this pipes are build with an high rescission, so it allows us to verify our results by fitting a cylinder with the known diameter into this pipes. Furthermore we plan to extend the structure and mount some plates with defined holes and slits on the backside and a big stopcock on front.

\subsection{Experiments}

Fig. 7 left shows our underwater 3D laser line scanner placed in the water tank during the calibration process. The scanner is mounted on a tripod and is pointed at a $3 \mathrm{D}$ calibration target 


\begin{tabular}{l|r} 
Components & Price in $€$ \\
\hline Roll-on/Roll-off container & 8460 \\
Scaffolding, including a staircase & 2480 \\
Oase BioTec ScreenMatic ${ }^{2}$ Set 90000 & 2222 \\
Oase LunAqua Power LED XL 4000 Wide Flood & 580 \\
$3 \mathrm{~m} \times 6 \mathrm{~m}$ tent & 380 \\
Hoarding & 830 \\
Small components & 1270 \\
& \\
\end{tabular}

Table 1. List of used components and their prices. The small components includes wooden planks, screws, wire and similar.

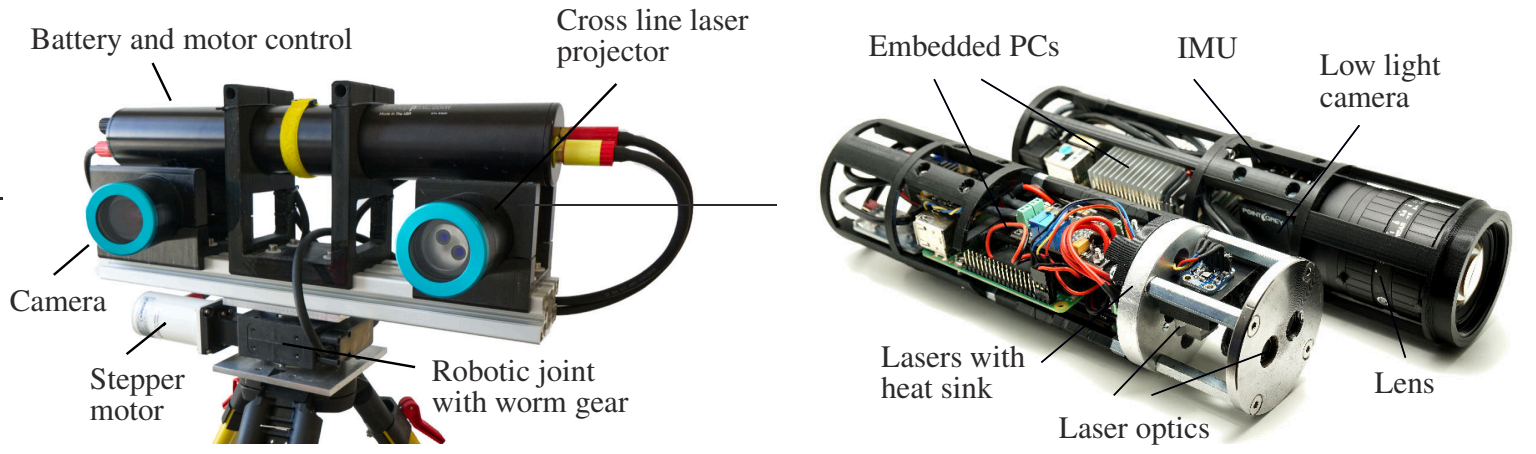

Figure 4. Structured light underwater laser scanner. Left: Scanner with motorized robotic joint mounted on a tripod, right: Detail view of the camera and laser projector assemblies mounted inside the underwater housings.
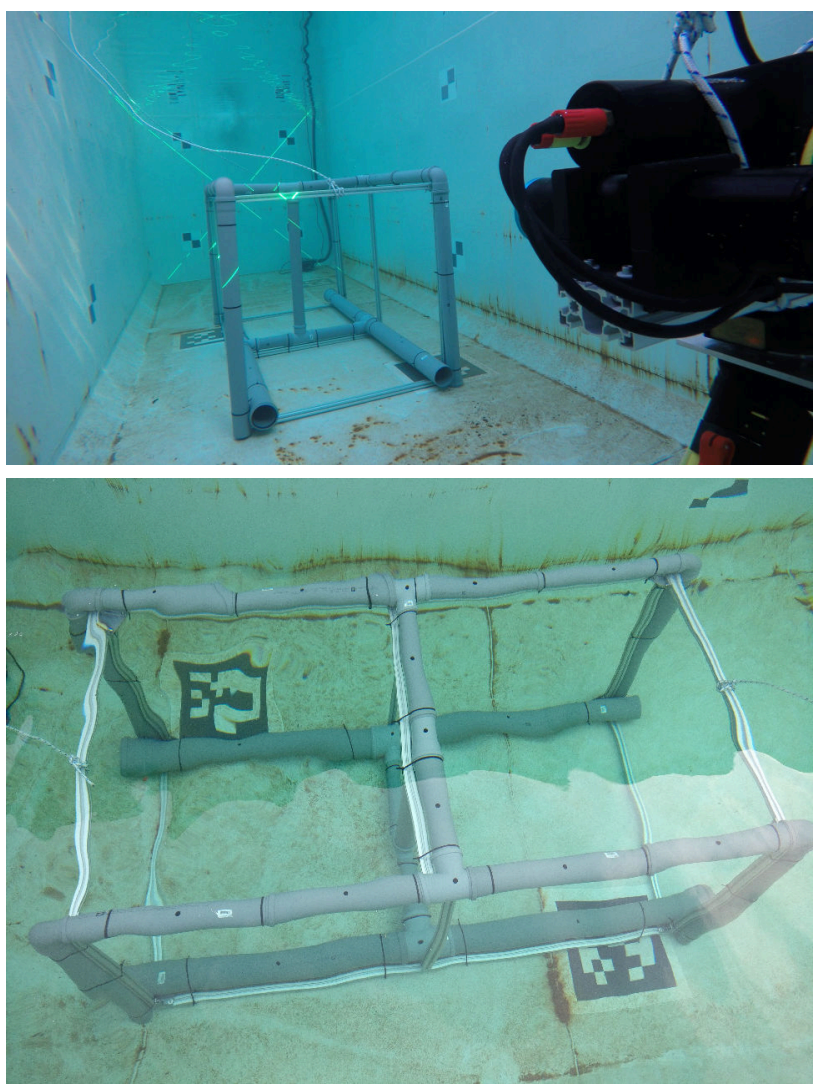

Figure 5. The first test structure placed in our water container. The size is $1 \mathrm{~m} \times 1 \mathrm{~m} \times 2 \mathrm{~m}$ and was build from item profiles and pipes.

with AprilTags. The calibration pattern was built from two glass plates, which are attached at a $90^{\circ}$ angle to each other. The AprilTags were printed directly on the glass plates to create calibration fixtures with high accuracy. The right picture in Fig. 8 shows the resulting point cloud of a scan captured in the container. This is an good example why an fully controllable environment is better than natural waters. In our container it is so much easier to capture good data with an defined setup for calibration. Fig. 7

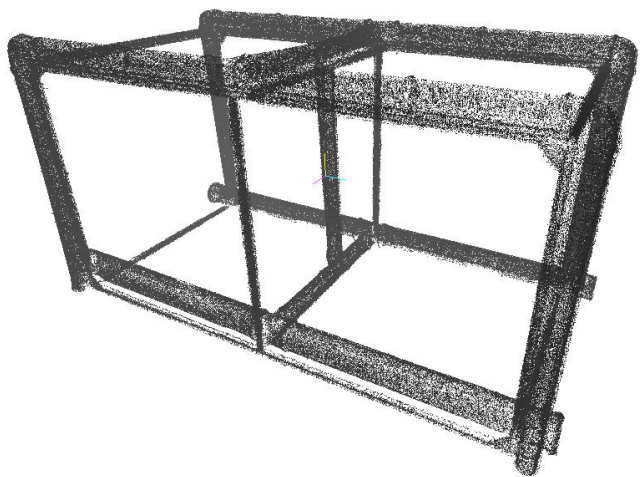

Figure 6. An 3D scan of the test structure, acquired by an Riegl VZ-400, as reference point cloud.

shows experiments for the verification of geometric models for underwater camera calibration.

Furthermore one of the first things we tried, was to scan the container it self. For this, we mounted our scanner on a tripod a acquired a full $360^{\circ}$ scan. Depending on the field of view of the scanner, it was not possible to scan the whole container at once. Therefore we would have to mount the scanner at different heights and register the resulting point clouds. This scans are depicted in Fig. 9. In Fig. 10 we registered the Riegl VZ-400 point cloud, the yellow one, against the data captured with the underwater scanner. The errors are very small and the data fits good together. However, the underwater scanner has only detected the lower part of the container so that the deformation of the container at the upper edge can no longer be seen in the scan.

\section{LESSONS LEARNED}

During the process of assembling all components of the environment and after filling it up with water, we realized that we had forgotten to make two holes for the hose of the pump and the return of the filter system into the container. So it was hard to drill 

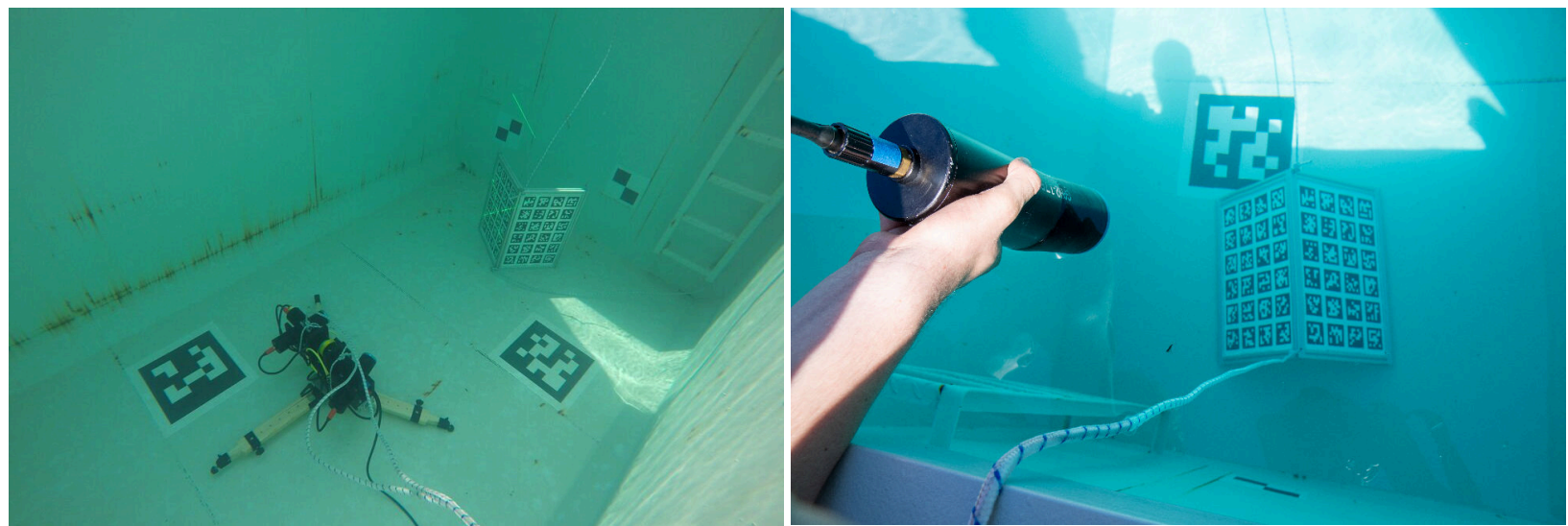

Figure 7. Left: One of the first experiments in the new test environment with our underwater 3D laser line scanner mounted on top of a tripod. Right: Experiments in the test environment for validation of refractive underwater camera models.

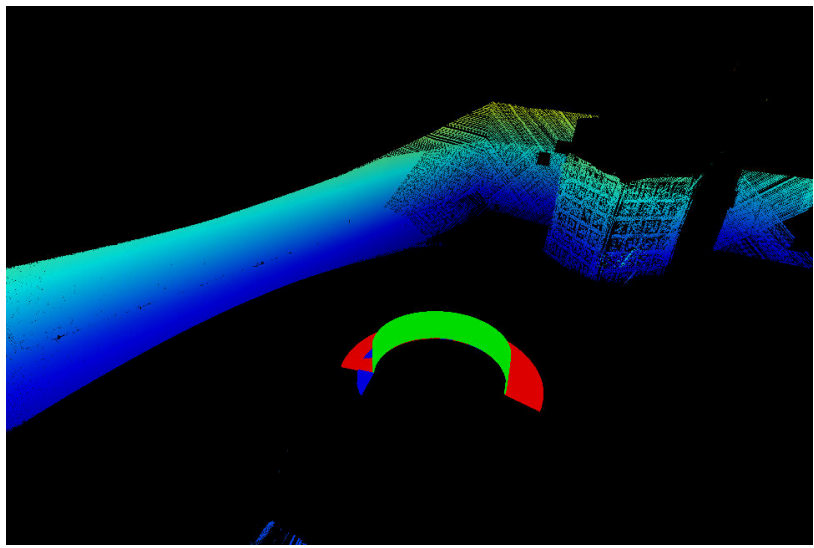

Figure 8. A point cloud of the L-shaped calibration pattern acquired with the 3D laser line scanner.

it throw the $5 \mathrm{~mm}$ steal walls without polluting the water. Had we selected the filter system before ordering the container, the manufacturer would have been able to attach two connection points for the hoses directly to us, and we would not have had to drill them later.

Another issue is, that the paint job was not perfect a lot of rust has formed along the welds. To protect the container from rust, it is necessary to install some magnesium anodes. These are very difficult to install in retrospect, as they must sit below the water surface and must be firmly connected to the metal of the container. Therefore, during the winter we will drain the water from the container, remove all rust, attach some anodes and repaint it. Therefore we must refill it with water in the spring.

Moreover, we underestimated the forces resulting from the enormous amount of water and did not consider that the container could deform there. Although this has no influence on the stability of the container, as it was specially reinforced for the loads occurring, but all the measuring marks on the outer walls became more or less useless. Also, the measurement of these brands and the previous scanning of the container as a reference has thus become obsolete. Therefore, we are currently building a precise specimen, which is then sunk in the water and is not deformed by the water pressure.

\section{CONCLUSION}

The goal was to build an low cost underwater test environment for less than $€ 20000$. Overall, our construction cost $€ 16222$ and is thus well below the planed bugedts and it's a lot cheaper to build than a solid pool. In addition, virtually no maintenance costs arise, these are limited to the running costs for the power of the filter system.

Apart from a few small things that we had to and still have to improve, we are very happy with the construction and would choose this setting again. The filter system makes a great job and after five month, the water is as clear and fresh as the first day. If the container is rusted and leaking in a few years, you have several options. Either you can simply mend the appropriate places or sell the container, at least to the scrap value of the steel, and for just $€ 8460$ a new container between the scaffold push.

Should a demonstration be carried out for a special occasion in another location, the container can be used in a few hours and set up for a few hundred euros with a truck at any location and put into operation. Just as easily, the whole area can be completely restored with little work. However, most things, such as the container, the fence and the scaffolding with stairs can be sold again at a good price.

All in all, we have created an excellent test environment for us, which makes it easier for us to carry out our future experiments. The only major upgrade we may make in the future is to equip the container with a crane. This would allow us to easily lift large objects into the water and possibly move test equipment with the crane in or above the water.

In addition, we work on different specimens to have a small selection of defined reference objects. These should enable us to objectively and repeatedly evaluate and assess the quality and accuracy of our scans and experiments.

\section{ACKNOWLEDGMENTS}

A big thank you goes to Jürgen Schemmel from the state fire brigade school Würzburg for help with filling up the tank. 
The International Archives of the Photogrammetry, Remote Sensing and Spatial Information Sciences, Volume XLII-2/W17, 2019 6th International Workshop LowCost 3D - Sensors, Algorithms, Applications, 2-3 December 2019, Strasbourg, France
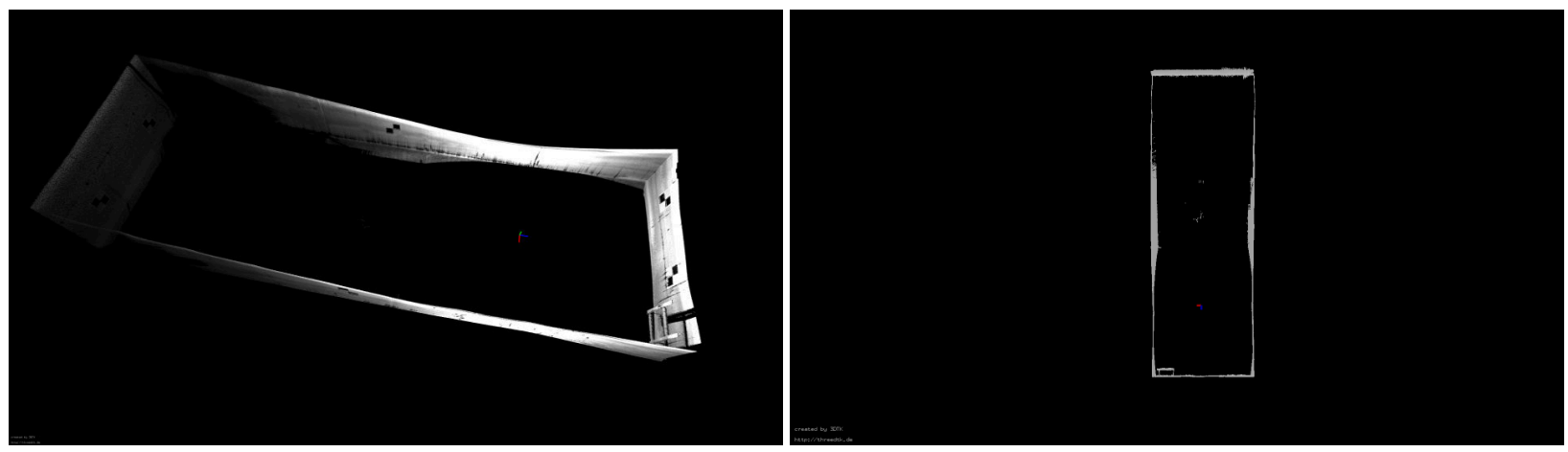

Figure 9. One of the first scans with our 3D laser line scanner in the test environment. On the right site is an top view to the point cloud.

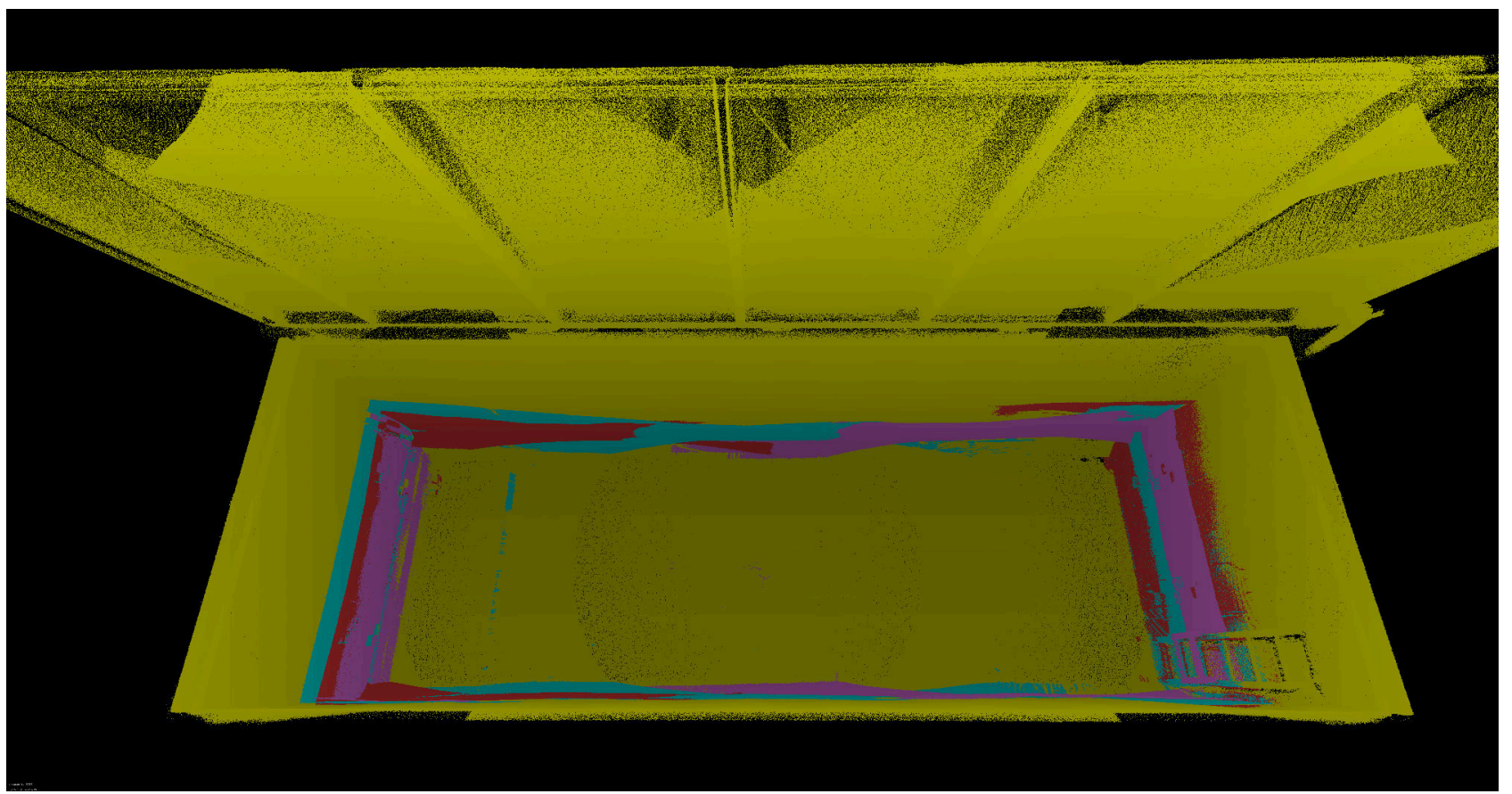

Figure 10. The point clouds of the container. The yellow one is acquired with the Riegl VZ-400 and serves as ground through, the others are acquired with the underwater scanner. It can be seen that the errors are very small.

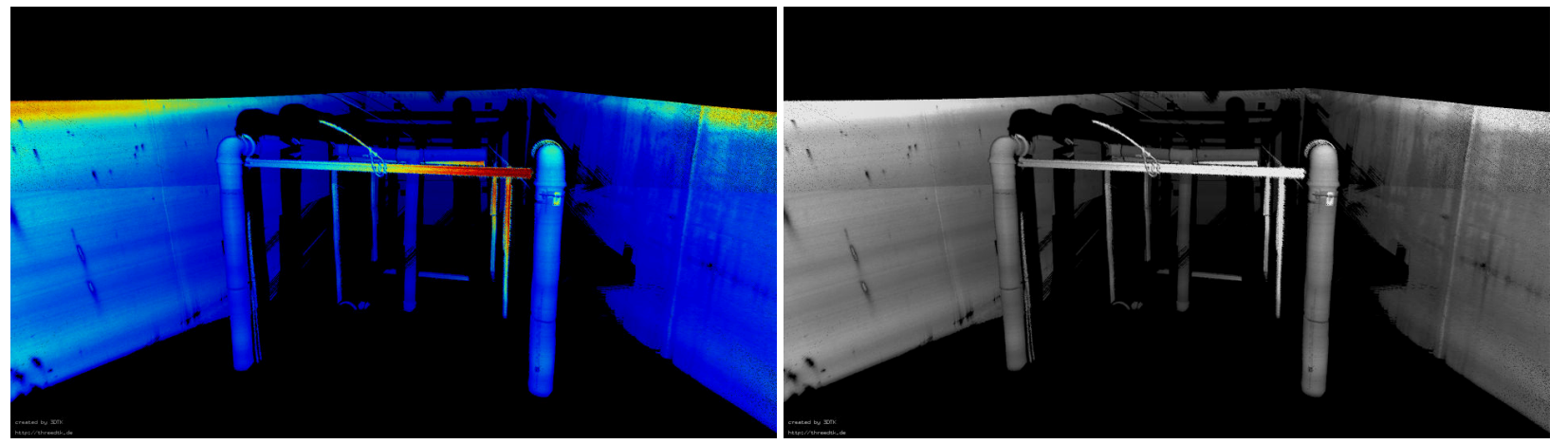

Figure 11. First underwater point cloud of the pipe test structure acquired with the 3D laser line scanner. 